Pre-print:

Working paper posted after two rounds of peer review (currently: R\&R). Last update: March, 2023.

All conclusions are preliminary.

\title{
The best-case heuristic: \\ Relative optimism in relationships, politics, and a global health pandemic
}

\author{
Hallgeir Sjåstad ${ }^{1}$ and Jay Van Bavel ${ }^{2}$ \\ ${ }^{1}$ Norwegian School of Economics \\ ${ }^{2}$ New York University
}

\begin{abstract}
In four experiments covering three different life domains, participants made future predictions in what they considered the most realistic scenario, an optimistic best-case scenario, or a pessimistic worst-case scenario ( $\mathrm{N}=2,900$ Americans). Consistent with a best-case heuristic, participants made "realistic" predictions that were much closer to their best-case scenario than to their worstcase scenario. We found the same best-case asymmetry in health-related predictions during the COVID-19 pandemic, future relationship satisfaction, and a future presidential election. In a fully between-subjects design (Experiment 4), "realistic" and best-case predictions were practically identical, and they were both faster than the worst-case predictions. These findings suggest that people might unknowingly be generating realistic scenario predictions $a$ s if they are making a bestcase prediction, indicating attribute substitution as part of the mechanism. Although political conservatism was correlated with lower covid-related risk perception and lower support of early public-health interventions, the best-case heuristic was ideologically symmetric.
\end{abstract}

Keywords: prediction; risk; optimism; heuristics; covid-19; political identity

\section{Open Science Practices}

Experiment 1 was non-registered. Experiments 2, 3 and 4 were pre-registered in advance of each data collection. Experiment 1 and 3 were based on convenience samples (Mechanical Turk platform), whereas Experiment 2 and 4 was conducted in samples that are nationally representative on the factors of age, gender, and ethnicity (Prolific platform). The Appendix, data and study materials for all four experiments are openly available:

https://osf.io/7xcqp/?view_only=8f30cd030da04d649788cf8093ffe39a. 


\section{The best-case heuristic: Relative optimism in relationships, politics, and a global health pandemic}

As we write this, the novel Coronavirus (COVID-19) has spread across all continents in the world, infecting more than 600 million people and taking more than 6.5 million lives in less than three years (WHO, 2022). According to public health experts this is the worst pandemic since the "Spanish flu" a century earlier (1918-1920). In the context of such a massive public health crisis, the accuracy and distribution of risk predictions can have direct consequences for both the individual and societal response, although perceptions of risk are not always as closely linked to reality as we think (Van Bavel et al., 2022). If people are overly optimistic on behalf of themselves compared to others, for instance, or if they only consider their best-case scenario for the future despite the dangers of a worst-case scenario, those expectations could serve as a justification for ignoring public-health advice and increasing the chances for infecting others.

The research in this paper was initiated during the first wave of the covid pandemic, in the early months of 2020, to develop a better understanding of how people make real-life risk predictions in such a difficult situation. We also examined the potential role of opposing political identities in shaping these predictions, and whether risk predictions were associated with support of life-saving public-health policies. Since then, we have expanded our scope to study future predictions across a broader set of different life domains, moving beyond the covid context to also include romantic relationships and political elections, to assess the generality of our findings. Across these diverse domains, we tested the general hypothesis that even when people are trying to be as realistic as they can, they tend to lean towards their best-case scenario when making future predictions. As our primary contribution, termed the best-case heuristic, we tested this hypothesis across four experiments and a total of 2,900 participants in the United States.

In four experiments, people predicted how likely it was that a specific future event would happen in the "most realistic" case, where some groups were randomly assigned to make similar predictions in either an optimistic best-case scenario or a pessimistic worst-case scenario. This design enabled a test of whether people would place their most realistic prediction in the middle of the best-case and worst-case estimates, reflecting an averaging strategy that perhaps a reasonable person would prefer, or alternatively, whether their "realistic" prediction would be more similar to their best-case scenario. Ranging across covid-related predictions of infection risk and likely waiting times for a new vaccine, to the chances of being in a happy relationship and being on the winning team after a future presidential election (US), we found robust and consistent evidence for the best-case heuristic - a novel type of relative optimism. Despite lower covid-related risk perception among conservatives than liberals, the general tendency to conflate one's most "realistic" prediction with a personal best-case scenario was equally strong on both sides of the political spectrum.

\section{The Psychology of Optimism}

In the first wave of the covid pandemic, no one could know for sure whether they would get infected, whether their friends and loved ones would remain safe, or how the pandemic would ultimately end. In situations like this, people are forced to rely on predictions, and these predictions might play an important role in our social cognition and public health behavior. It is therefore a central challenge both for social scientists and policy-makers to understand how such predictions are made, and how differences in risk perception might be connected to pandemic responses and political identities in the wider public (Van Bavel et al., 2020).

Prior research from psychology has documented optimistic bias in a wide range of different settings (Alicke, 1985; Taylor \& Brown, 1988; Sedikides \& Gregg, 2008; Tappin \& McKay, 2017). In particular, people are prone to make favorable predictions of the future (Rogers et al., 2017; Sjåstad et al., 2022), and to do so in ways that sometimes exceed base-rate 
frequencies or social standards for comparison (Weinstein, 1980; Cooper et al., 1988; Jansen et al., 2011; Rothman \& Klein, 1996; Waters et al., 2011). The tendency to overestimate the probability for positive outcomes and underestimate personal risk, has traditionally been referred to as "unrealistic optimism" (for reviews, see Shepperd et al., 2013; 2015; for a critical view, see Harris \& Hahn, 2011; Krizan \& Windschitl, 2007). The judgments studied include topics like personal health, social relationships, and financial planning. When asked to list important life events that could happen in their future, people reported more desirable than undesirable events, and the desirable events were also rated as more likely than unlikely (Hoorens et al., 2008). Studies on the "planning fallacy" have documented that people tend to underestimate the time needed to complete future tasks, such as academic papers or business reports, in which the average time prediction is unrealistically close to what you would expect in a statistical best-case scenario (Buehler et al., 2010; Tversky \& Kahneman, 1974). Building on this research, we reasoned that people might also be leaning towards their own best-case scenario when trying to make realistic predictions, and that this optimistic asymmetry could manifest even in the context of personal risk assessment in a global pandemic, and when evaluating the chances for happy romantic relationships and a favorable election result.

Yet, it could also be the case that these rosy prediction patterns are tempered by a more sober assessment during a global health crisis, like the current COVID-19 pandemic, when the stakes are high and people are frequently exposed to vivid information about real danger. Indeed, it has already been found that future optimism is far from universal in human judgment. For instance, research on the "hard-easy effect" has found that people tend to be optimistic for easy tasks but are pessimistic for difficult tasks (Keren, 1991; Erev et al., 1994; Moore \& Healy, 2008). Relatedly, common events are often overestimated whereas rare events, including positive ones, are underestimated (Chambers et al., 2003; Kruger \& Burrus, 2004; Harris et al., 2017). Thus, future optimism may often occur but not always. It is therefore an open question to what extent people will express "relative optimism" in the current research, by leaning towards a personal best-case scenario in the domains of covid, romantic relationships, and a future presidential election.

\section{Types of Optimism}

When the true probability of a specific outcome is either unknown or highly uncertain, one way people might sustain a positive view of the future is to construe optimistic expectations by comparison. This can be done by imagining that they will be better off than other people, known as "comparative unrealistic optimism" at the group level (Shepperd et al., 2013; 2015. It can also be done by assuming that their "realistic" risk or most likely outcome is much closer to their own best-case scenario than to their worst-case scenario. The current experiments were designed to test both forms of relative optimism, in which the first is well-established and the second is more novel. We are particularly interested in these types of predictions as they are inherently social, drawing on assumptions about other people and eliciting specific person perceptions in return (e.g., Hoorens et al., 2017). Indirect measures of risk also appear to account for unique variance in concern and behavioral intentions (e.g., Klein, 2002; Rose, 2010), as a supplement to general (non-comparative) measures of risk perception.

Self-other comparisons. Studies of so-called unrealistic optimism have found that people sometimes overestimate their chances for positive life events relative to others, and that they also believe to be personally less at risk for negative outcomes (Shepperd et al., 2013; 2015). These effects are usually described as comparative optimism, because people are asked to compare their own chances to their peers, either explicitly by providing a single comparative risk judgment, or indirectly, by asking respondents to provide two absolute risk judgments: one for themselves and one for the comparison others (Covey \& Davies, 2004). 
In this type of research, participants are typically asked to make a single likelihood prediction for each specific outcome. However, people can also think about the future in terms of multiple possibilities and alternative scenarios (Baumeister et al., 2018; Phillips et al., 2019; Redshaw \& Suddendorf, 2016), not just as a straight path or a single point estimate. When facing a pandemic, even public health experts tend to think in terms of different scenarios that may or may not happen (e.g., IHME, 2020). In our view, it is an open question whether classic comparative optimism in judgments of oneself versus others will be equally prevalent across an extended range of alternative scenarios, such as when participants are instructed to consider a worst-case scenario where things presumably did not work out as planned. Will the person still assume to be at lower comparative risk than other people? As such, the present experiments extend previous research by obtaining risk predictions for oneself and others in a "worst-case scenario" and a "best-case scenario", in addition to their "most realistic" estimate.

Best-case heuristic. The relative position of the three estimates (realistic, best-case, worst-case), might reveal a second facet of optimism, namely what we refer to as the best-case heuristic. When people generate what they see as realistic predictions, they might lean towards their own best-case scenario for the future, and in comparison, place less weight or fail to consider their worst-case scenario to the same extent. In the context of the early stage of the COVID-19 pandemic, a pattern of best-case optimism could have severe consequences if increased risk-taking lead people to contract the virus and then potentially pass it on to others. In the domains of romantic relationships and politics, neglecting the possibility of a worst-case scenario might leave the person less prepared for future obstacles and pitfalls if they should occur. At a theoretical level, a pattern of best-case optimism could provide interesting insights into the cognitive process of generating both "realistic" and alternative scenarios for the future.

Yet, few studies appear to have examined the possibility of a best-case asymmetry of this kind. In one rare exception, people in a study of the planning fallacy were asked to indicate their best and worst scenario for how much time they would need to complete a school assignment and their tax income return (Newby-Clark et al., 2000). This research found that best-case scenarios appeared more plausible than worst-case scenarios, and that people largely disregarded the worst-case scenario when predicting their task completion time. Another study (Tanner \& Carlson, 2009) found that standard predictions of future behaviors (blood donation, exercise frequency, saving for retirement, and completing an assignment) were almost indistinguishable from predictions of the same behaviors in an "ideal world". However, worst-case estimates were not included in this design.

We note that the participants in neither of these studies received any instruction to be as realistic or accurate as they could in the baseline condition. This is an important detail, as a recent study found that a simple accuracy induction can reduce cognitive bias in information evaluation (Bago et al., 2020). Thus, without explicit instruction, it remains unclear to what extent participants tried to be as realistic as they could when they expressed an overly optimistic view of the future. We therefore included this instruction in all our experiments. Seen as a whole, we consider the previous research as suggestive, but insufficient, evidence for a best-case heuristic in risk estimates and future predictions. In our view, it would be more telling if what people believe is their most realistic scenario turns out to be rather optimistic when compared to their own alternative predictions. This would be particularly striking if it happened in a domain where the risk involves life or death consequences - where the accuracy incentives are high. To examine whether the best-case heuristic is a domain-general pattern in human judgment, we will also move beyond the COVID-19 context to study similar predictions about romantic relationships and a future presidential election.

The idea of a best-case heuristic is based on a general understanding of heuristics as an effort-reducing simplification strategy (Shah \& Oppenheimer, 2008), and suggestive evidence 
that what people want comes first to mind when predicting the future (Hoorens et al., 2008; Sjåstad \& Baumeister, 2022). Specifically, we propose that people's best-case scenario often represents a natural default or anchor for their prediction (Tversky \& Kahneman, 1974), which creates a more positive starting point for their "realistic" estimate than the midpoint between their best and worst alternative scenarios. When studied in fully between-subjects designs that only ask participants to consider one scenario (Experiment 4), "realistic" and best-case predictions might even be statistically undistinguishable from each other, suggesting an underlying process of optimistic attribute substitution (Kahneman, 2003). That is, people might unknowingly answer the question of what is most realistic with a best-case prediction of what they hope will happen, because the goal-consistent best-case scenario comes first to mind.

Interestingly, the best-case heuristic implies that people do not naturally adhere to the successful bias-reducing strategy of averaging across multiple estimates to "harness one's inner crowd" when estimating unknown quantities (van Dolder \& van den Assem, 2018; Herzog \& Hertwig, 2009; Stroop, 1932). Instead, we might find that the person is guided by a one-sided heuristic that places systematically greater weight on the positive end of the internal probability distribution. Would the lay person consider this a good decision strategy? According to our own belief survey, reported in the Appendix, the predicted best-case asymmetry would not only be in conflict with the simple averaging strategy reviewed above. It would also be in conflict with normative lay beliefs. When considering the current experimental setting from a third-person outside perspective, a majority of a nationally representative US sample $(N=467)$ believed that a "reasonable person" would typically opt for the middle between the best-case and worst-case scenarios (domain-general predictions: $59 \%$, relationship-specific predictions: $50 \%$ ), if trying to be as realistic and accurate as possible. To be sure, these results cannot say anything about what prediction strategy actually performs best in real life. But as a lay-belief normative standard, the survey do suggest that many people believe that opting for the middle will tend to be the best prediction strategy in such situations.

\section{Risk Prediction, Political Identity, and Policy Support}

Understanding why people comply or ignore public health advice is critical during a pandemic and other health crises (Van Bavel et al., 2020; Capraro et al., 2021). During the H1N1 pandemic in 2009, one study found that risk perception was positively correlated with compliance to public health advice in the Netherlands (Bults et al., 2011). A study relying on hypothetical scenarios found that higher risk estimates were positively correlated with the willingness to use face masks during a pandemic and the willingness to get vaccinated (Barr et al., 2008). This research suggests that risk assessments might play an important role in public health behavior. In our most covid-oriented experiments (1-2), we therefore wanted to examine whether the predicted risk of infection (for oneself and others) would be positively correlated with the support of public-health covid interventions.

One potential barrier for coordinated public health behavior within countries, is group variation in political ideology - especially in polarized political contexts. There is some evidence that political conservatism is associated with a heightened attunement to threats and disgust sensitivity compared to liberals (Inbar et al., 2009), and conservatives were more concerned about being exposed to Ebola during the epidemic in 2014 (NW et al., 2014). However, several studies have been unable to replicate the relationship between disgust sensitivity and political conservatism (e.g., Tybur et al., 2010), and recent work has also questioned the association between political conservatism and domain-general threat perception (Fiagbenu et al., 2021).

Alternatively, the psychology of partisan identity could provide the exact opposite pattern of results (Van Bavel \& Pereira, 2018). In contexts where there is strong 'affective polarization', in which partisans dislike and distrust those from the opposing party(ies), people from either party often believe partisan content over public policy information, and may even be more 
susceptible to believing false information (Iyengar et al., 2019; Mason, 2018). In the current setting of the US, there has been a notable partisan divide in concern for the pandemic and appropriate policy responses, in which republican leaders on the right have appeared to be less concerned than democratic leaders on the left, which has been mirrored in polling results as well (Van Bavel, 2020; Kushner Gadarian et al., 2020). During the early stage of the pandemic, people from more conservative or Republican parts of the United States were less likely to follow social distancing guidelines, and this was related to a subsequent increase in infections and mortality in those regions (Gollwitzer et al., 2020). We therefore examined whether high risk predictions would be associated with stronger support for public health lockdown policies, and whether right-leaning (vs. left-leaning) political views would be associated with lower risk estimates and lower support of early lockdown policies.

The research questions reviewed above, focusing on political identity and policy support, are concerned with potential correlations between absolute levels of predicted infected risk. In addition, we will also examine whether our primary hypothesis, the best-case heuristic, will be moderated by political ideology. It is an ongoing debate in psychology and social science whether political bias is symmetric, being roughly evenly distributed among conservatives and liberals (Ditto et al., 2019), or whether it is asymmetric, by being more frequently observed or stronger in magnitude among right-leaning conservatives (Altemeyer 1981; 1996; Jost et al., 2003; Eidelman et al., 2012). In the current research, we tested whether the tendency to conflate one's "realistic" predictions with a best-case scenario is equally common across the right-left political spectrum, or whether it is substantially stronger or perhaps only present at one of the sides. In our final experiment (Experiment 4), we will also test whether both sides of the rightleft political spectrum will be equally optimistic that their own party will win the next presidential election in the United States.

\section{Current Research}

We conducted a series of four experiments, testing our primary hypotheses on a total sample of about 2,900 participants from the United States. In experiments 1 and 2 (March 2020), people predicted how likely it was that they would become infected by COVID-19 by the end of the year (i.e., 9 months into the future). In Experiments 3 and 4 (April 2020 and September 2022), we used a similar scenario method to examine future expectations in different settings. This included predicted waiting times for a publicly available covid vaccine, predicted relationship satisfaction five years into the future, and predictions of the most likely outcome of the next US presidential election.

The primary hypothesis is that people will express two different kinds of relative optimism in their risk estimates and future predictions. First, we predicted that participants would consistently estimate their own infection risk as lower than the risk for the "average person". This hypothesis is derived from previous findings of comparative unrealistic optimism (Shepperd et al., 2015; Weinstein, 1980). Our experiments aimed to examine whether this egocentric bias will generalize to predictions in alternative scenarios as well, including a best-case scenario and a worst-case scenario. As the second form of relative optimism, we predicted that participants would rely on a best-case heuristic, meaning that their "realistic" estimates are systematically closer to their best-case scenario than to their worst-case scenario. Importantly, we will test the best-case heuristic across three different prediction domains: the covid pandemic, future relationship satisfaction, and a future presidential election.

Turning to the potential role of political identity and our secondary hypotheses, we expected that participants' general risk estimates in the first wave of the covid pandemic would be negatively correlated with right-leaning conservative (vs. left-leaning liberal) political views and positively correlated with support of anti-pandemic policies (e.g., physical distancing and early lockdown interventions). Finally, we examined whether the domain-general reliance on the 
best-case heuristic is ideologically symmetric, or whether it only occurs at one of the two sides of the right-left political spectrum. For exploration, we will estimate the statistical accuracy of "realistic" predictions in the absolute sense as well, by comparing the subjective probability estimates (0-100\%) made by participants to representative base-rate data. These base-rate comparisons were not guiding the design of the current experiments, which focus on relative optimism, and were not pre-registered or predicted a priori. We have decided to report these comparisons throughout the paper nonetheless, as exploratory analyses of optimism versus pessimism in the absolute sense, since they are of general interest.

We report a sensitivity power analysis for the sample size in each experiment, all data exclusions, all manipulations, and all measures. Data and study materials for all four experiments are openly available: https://osf.io/7xcqp/?view only=8f30cd030da04d649788cf8093ffe39a.

\section{Experiment 1: Relative Optimism in Risk Prediction}

Experiment 1 was a non-registered online experiment, conducted in USA during the first wave of the pandemic (25th of March 2020). At this point, the incidence of COVID-19 had exceeded one million confirmed cases and claimed more than 100,000 lives worldwide, with numbers rising rapidly in the US (United States Coronavirus, Worldometer). Focusing on predicted risk for virus infection by the end of the year (2020), we recruited 330 American participants from a convenience sample at Amazon's Mechanical Turk. After participants who failed a simple attention check were excluded, the final sample consisted of 308 American participants $\left(M_{\mathrm{age}}=41\right.$ years, $46 \%$ women). A sensitivity analysis in the $\mathrm{G}^{*}$ power software showed that the final sample size $(N=308)$ provided statistical power corresponding to an $80 \%$ chance to detect a main effect of $d=0.32$ or larger ( $p<.05$, two-tailed).

The participants were instructed to provide their "most realistic" prediction of how likely it was to become infected by the Coronavirus by the end of the year (i.e., 9 months into the future), both for themselves and the average person in separate judgments. They were then randomly assigned to provide similar predictions in what they considered their optimistic bestcase scenario or their pessimistic worst-case scenario. All risk predictions were made on a similar scale ranging from $0-100 \%$ likely. After providing their risk predictions, all participants responded to a broad set of attitude measures, including support of public-health policies (e.g., early lockdown and travel restrictions) and political orientation.

Procedure and measures. First, all participants read a brief text about the outbreak of the Coronavirus, describing common responses and symptoms, but without any information about specific infection rates or probabilities. On the next screen, participants were instructed to provide their most realistic prediction of infection risk by the end of the year, both for themselves and others. Headline: "In the most realistic case, by December 31, 2020:". Specific items: "How likely do you think it is that you will get infected by the Coronavirus (Covid-19)?" (0-100\% likely), and "How likely do you think that the average person in the US will get infected by the Coronavirus/Covid-19?" (0-100\% likely). The response scale for each probability judgment increased in increments of 10.

After reporting their "most realistic" predictions, on the next screen participants were randomly assigned to provide similar predictions in their best-case scenario or their worst-case scenario. Best-case headline: "In the best-case scenario (an optimistic view), by December 31, 2020:". Worst-case headline: "In the worst-case scenario (a pessimistic view), by December 31, 2020:". The wordings of the specific prediction items were identical as in the realistic predictions (both for self and the average person), only the headline instructions above the items varied between conditions.

After providing their risk predictions, the participants responded to a set of attitude measures, which included policy support and political orientation. Policy support: Five items asked to what extent participants supported different lockdown policies for the next month: 
mandatory shutdown of all kindergartens, schools, and universities; bars and restaurants; public gatherings; international travel; restrictions of non-essential outdoor movement; all to be rated from $1=$ strongly oppose, to $4=$ strongly support. Political orientation was measured with a single item commonly used in previous research: "In general, what would be the best description of your political views?" ( $1=$ very liberal/left-leaning, $4=$ centrist/moderate, $7=$ very conservative/right-leaning).

\section{Primary Hypotheses: Relative Optimism}

\section{Results}

In the realistic scenario, participants predicted that it was on average $37.6 \%$ likely $(S D=24.8)$ that they would become infected by the Coronavirus by the end of the year, against $44.5 \%(S D=$ 23.2) for the "average person". In the best-case scenario, they predicted that it was $22.4 \%$ likely $(S D=23.2)$ for themselves against $29.9 \%(S D=22.7)$ for the average person. In the worst-case scenario, they predicted that it was $59.4 \%$ likely $(S D=27.9)$ for themselves and $64.4 \%$ likely $(S D=25.5)$ for the average person.

In line with our first hypothesis, the risk estimates were lower for oneself than the average person in all three scenarios, as displayed in the upper half of Table 1. The self/other differences were highly significant and the effects were quite strong (realistic case: $t(307)=8.33$, $p<.001, d=.48$; best-case: $t(151)=6.51, p<.001, d=.53$; worst-case: $t(155)=4.23, p<.001$, $d=.34$ ). This provided a successful replication of the classic form of "comparative unrealistic optimism", which also extended to best-case and worst-case estimates.

In line with our second hypothesis about a best-case heuristic, the "realistic" predictions were closer to the participants' best-case scenario than to their worst-case scenario, as shown in the two last columns of Table 1. Specifically, when calculating a difference score between the realistic prediction and each of the two alternative predictions, the distance between realisticoptimistic was consistently smaller than the distance from realistic-pessimistic. This difference was statistically significant both for predictions about oneself $(t(306)=3.30, p=.001, d=.38)$ and the average person $(t(306)=2.67, p=.008, d=.30)$. The strength of the effects was moderate but robust. These results suggest that people are "relative optimists" not only when comparing themselves to other people, but also when comparing their attempt at realistic prediction to their own alternative scenarios, in which they were leaning towards a best-case scenario for the future.

\begin{tabular}{llllll}
\hline & Realistic & Best-case & Worst-case & Best diff & Worst diff \\
\hline Experiment 1 & & & & & \\
$\quad$ Self & $37.6 \%(24.8)$ & $22.4 \%(23.2)$ & $59.4 \%(27.6)$ & -15.2 & 21.8 \\
$\quad$ Average person & $44.5 \%(23.2)$ & $29.9 \%(22.7)$ & $64.4 \%(25.5)$ & -14.6 & 19.9 \\
Experiment 2 & & & & & \\
$\quad$ Self & $36.0 \%(25.1)$ & $22.2 \%(23.0)$ & $59.4 \%(27.9)$ & -13.8 & 23.4 \\
$\quad$ Average person & $44.6 \%(23.5)$ & $31.1 \%(21.9)$ & $66.3 \%(24.7)$ & -13.5 & 21.7 \\
& & & & & \\
\hline
\end{tabular}

Table 1: Relative optimism in Experiment 1 and 2

Mean risk predictions of getting infected by the Coronavirus by the end of the year in the US (2020). Predictions were made for oneself and others ("the average person") in realistic, best-case, and worst-case scenarios on 11point probability scales from 0 to $100 \%$ (SD in parentheses). Difference scores in the two last columns show mean distances from realistic estimates to best-case and worst-case predictions, respectively. The infection rate was consistently rated as lower for oneself than others, and the "realistic" predictions were positively skewed, meaning that the realistic predictions were closer to the best-case scenario than to the worst-case scenario. 


\section{Secondary Hypotheses: Politics and Policy Support}

In the correlational analysis, focusing on the role of political identity and policy support, we found that the "realistic" baseline predictions that participants made of their future infection risk were positively associated with their support of COVID-19 public-health policies $(r=.17, p=$ .003 ). That is, people who evaluated the probability for future infection as relatively high (vs. low), on average expressed stronger support of early lockdown and other policy interventions to reduce the spread of the virus. We note that the strength of this association was relatively weak.

As for the role of political orientation, right-leaning conservatives (vs. left-leaning liberals) predicted lower infection risk for others $(r=-.20, p=.002)$, but not for themselves $(r=-.10, p=$ $.121)$, and they were also less supportive of public-health lockdown policies $(r=-.35, p<.001)$. However, a majority in both political groups expressed moderate or strong support of all five lockdown interventions they were presented for (conservatives: 73\%, liberals: 84\%).

\begin{tabular}{|c|c|c|c|c|c|}
\hline & & Self: realistic risk & Other: realistic risk & Covid policy support & Political conservatism \\
\hline Self: realistic risk & $\begin{array}{l}\text { Pearson's r } \\
\text { p-value }\end{array}$ & - & & & \\
\hline Other: realistic risk & $\begin{array}{l}\text { Pearson's } r \\
p \text {-value }\end{array}$ & $\begin{array}{l}0.818 \cdots \\
<.001\end{array}$ & - & & \\
\hline Covid policy support & $\begin{array}{l}\text { Pearson's r } \\
\mathrm{p} \text {-value }\end{array}$ & $\begin{array}{l}0.171^{* *} \\
0.003\end{array}$ & $\begin{array}{l}0.238 \cdots \\
<.001\end{array}$ & - & \\
\hline Political conservatism & $\begin{array}{l}\text { Pearson's r } \\
\text { p-value }\end{array}$ & $\begin{array}{r}-0.101 \\
0.121\end{array}$ & $\begin{array}{l}-0.200^{* *} \\
0.002\end{array}$ & $\begin{array}{l}-0.345 * * \\
<.001\end{array}$ & - \\
\hline
\end{tabular}

Note. ${ }^{*} \mathrm{p}<.05,{ }^{* *} \mathrm{p}<.01,{ }^{* * *} \mathrm{p}<.001$

\section{Table 2}

Correlation matrix for Experiment $1(N=308$, Mechanical Turk sample, USA), showing statistical associations between four key variables in the context of the COVID-19 pandemic.

For exploration, we examined whether political orientation would moderate our primary finding of relative optimism. In contrast to that possibility, a moderation analysis in Jamovi found no interaction between experimental condition and political orientation when retesting the best-case heuristic hypothesis $(Z=-.93, p=.35)$. Despite the lower general risk predictions among right-leaning conservatives than left-leaning liberals, this explorative analysis suggests that the tendency to provide "realistic" predictions that are closer to one's best-case scenario than the worst-case scenario was equally prevalent on both sides of the right-left political spectrum.

\section{Experiment 2: Relative Optimism in Risk Prediction}

The results of Experiment 1 provided initial evidence of "relative optimism" in risk prediction, and suggested that conservatives saw the pandemic as a lower general risk. However, the sample was relatively small, making it hard to detect small and moderate effect sizes, and the hypotheses were not pre-registered. Therefore, Experiment 2 was a pre-registered, high-powered and direct replication (PDF: http://aspredicted.org/blind.php? $\mathrm{x}=\mathrm{s} 666 \mathrm{~h} 7$ ), recruiting 1250 American participants from a representative online sample at Prolific. This experiment was conducted on the 27th and the 28th of March (2020).

After excluding 11 participants who failed the attention check and 37 participants who reported that they had tested positive for COVID-19, the final sample consisted of 1202 attentive participants without COVID-19 $\left(M_{\mathrm{age}}=36\right.$, years, $49 \%$ women), which is about four times as large as the original sample in Experiment 1. The sample was nationally representative on the factors of age, gender and race/ethnicity. A sensitivity analysis showed that the final sample $(N=$ 1,202 ) provided statistical power corresponding to a $95 \%$ chance to detect a main effect of $d=$ 0.21 or larger $(p<.05$, two-tailed). Compared to much experimental research in psychology and behavioral science, this enables a reliable and highly informative test of the hypotheses. In 
comparison, a meta-analysis of 3,801 published papers in psychology and neuroscience (Szucs \& Loannidis, 2017) concluded that the median study only had $44 \%$ power to detect an effect of Cohen's $d=.50$ and $12 \%$ power to detect a smaller effect of $d=.20$.

Procedure and measures. Experiment 2 was a direct replication of Experiment 1. The only design difference from Experiment 1, was the addition of three exploratory attitude measures at the end of the survey. Of particular interest for the current paper, this included a belief measure of national pandemic preparedness: To what extent participants considered their own country (USA) to be better or worse prepared than other countries to reduce the spread of the virus and provide sufficient medical treatment during the first months of the pandemic $(-3=$ much worse, $0=$ equally prepared, $+3=$ much better). The two other measures focused on human nature beliefs and beliefs about future well-being (see Appendix).

\section{Primary Hypotheses: Relative Optimism}

\section{Results}

As their most realistic estimate, the participants in Experiment 2 predicted that it was on average $36.0 \%$ likely $(S D=25.1)$ that they would get infected by the Coronavirus by the end of the year (2020), compared to $44.6 \%(S D=23.5)$ for the average person. In the best-case scenario, they predicted that it was $22.2 \%$ likely $(S D=23.0)$ for themselves against $31.1 \%(S D=$ $21.9)$ for the average person. In the worst-case scenario, they predicted that it was $59.4 \%$ likely $(S D=27.9)$ for themselves and $66.3 \%$ likely $(S D=24.7)$ for the average person. Mean risks displayed in the lower panel of Table 1 show that all estimates are highly similar to those of Experiment 1, with significantly lower estimates for oneself than others in all three prediction scenarios (realistic: $t(1201)=18.01, p<.001, d=.52$; best-case: $t(600)=14.48, p<.001, d=$ .59 ; worst-case: $t(600)=9.75, p<.001, d=.40)$.

Consistent with the best-case heuristic, providing a direct and high-powered replication of Experiment 1, the distance from the "realistic" predictions to the best-case scenario was smaller than the distance from the "realistic" predictions to the worst-case scenario. This was true both for predictions about oneself $(t(1200)=9.73, p<.001, d=.56)$ and the average person $(t(1200)=9.09, p<.001, d=.53)$. The effects were relatively strong and highly significant. Thus, when participants were instructed to provide their most realistic predictions of future infection risk, they systematically leaned towards their best-case scenario. 
COVID-19:

Risk prediction for self

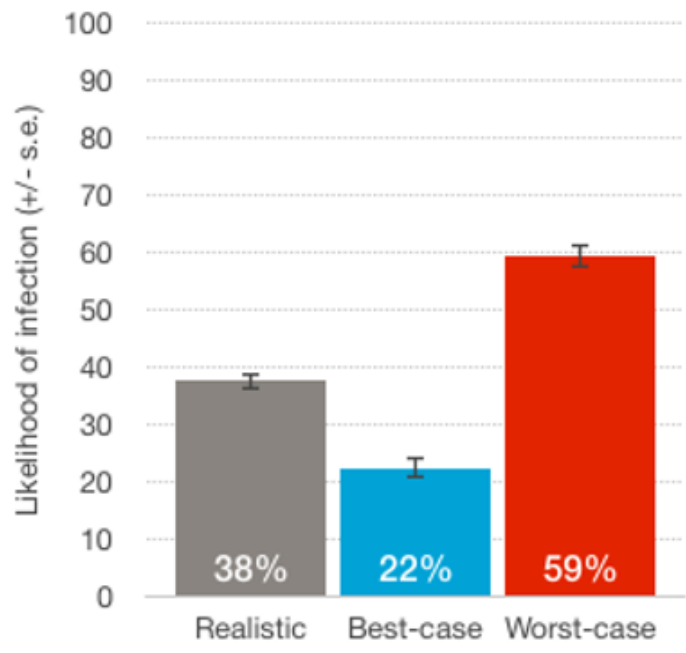

COVID-19:

Risk prediction for others

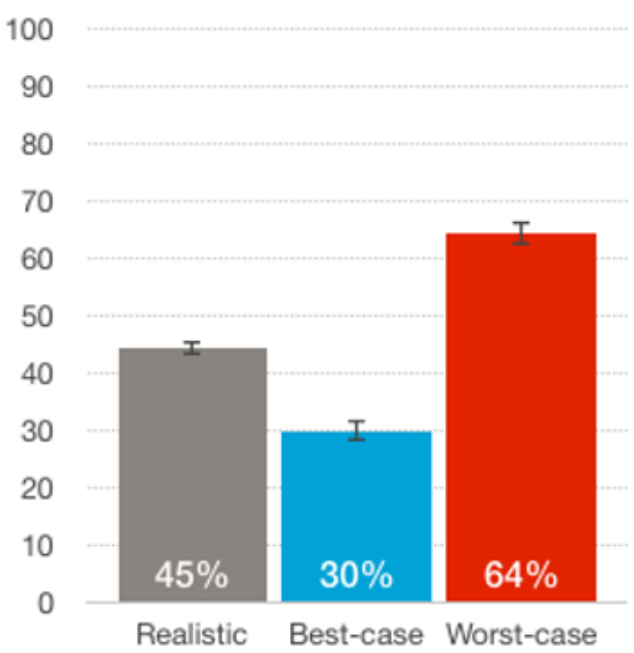

Figure 1: Relative optimism, Experiment 1

Experiment $1(N=308$, Mechanical Turk sample, USA): In line with a best-case heuristic, the "realistic" predictions were positively skewed both for oneself $(p=.001, d=.38)$ and the average person $(p<.008, d=.30)$. That is, when participants reported their most realistic estimate of how likely it was to get infected by the Coronavirus by the end of the year (2020), they were leaning towards their best-case scenario. Extending the classic finding of comparative optimism, risk estimates were also consistently lower for oneself than the average person in all three scenarios.

COVID-19:

Risk prediction for self

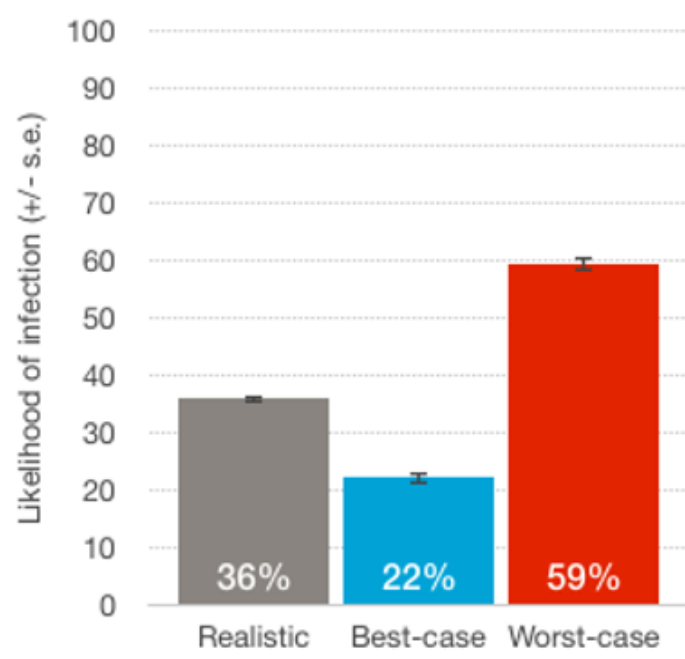

COVID-19:

Risk prediction for others

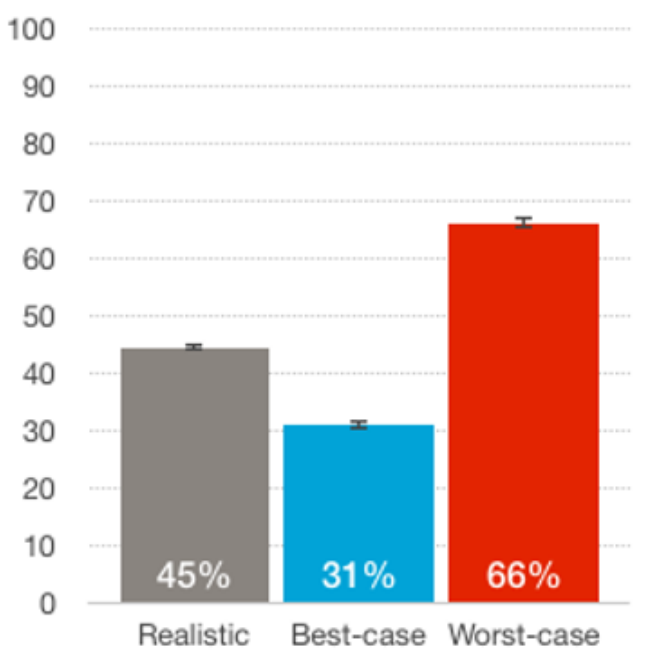

Figure 2: Relative optimism, Experiment 2

Experiment $2(N=1202$, Prolific representative sample, USA): Providing a direct replication of the best-case heuristic from Experiment 1, the "realistic" predictions were positively skewed both for oneself $(p<.001, d=.56)$ and the average person $(p<.001, d=.53)$, meaning that it was systematically closer to the best-case scenario than the worstcase scenario. Also replicating Experiment 1, risk estimates were consistently lower for oneself than the average person in all three scenarios. 


\section{Secondary Hypotheses: Politics and Policy Support}

Turning to the role of political identity and public-health policy support, the results showed a similar pattern as Experiment 1 . The "realistic" risk estimates were positively correlated with support of public-health policies $(r=.22, p<.001)$, meaning that people who evaluated the probability for future infection as relatively high, expressed stronger average support of restrictive lockdown policies during the early stage of the pandemic. Moreover, rightleaning conservatives (vs. left-leaning liberals) gave lower infection risk estimates both for themselves $(r=-.19, p<.001)$ and others $(r=-.24, p<.001)$, and were less supportive of early lockdown policies $(r=-.21, p<.001)$. Like before, a majority in both political groups expressed moderate or strong support to all five lockdown interventions in the policy support measure (conservatives: $88 \%$, liberals: 95\%). Taken together, these results provide a broad replication of the findings from Experiment 1 in a much larger, more representative sample of Americans using pre-registered methods and hypotheses.

Turning to the new explorative measure of belief in national preparedness during the early stage of the pandemic (i.e., general ability of reducing the spread of the virus and providing sufficient medical support to those in need), $68.3 \%$ of the participants thought the US was less prepared than other countries and $22.4 \%$ thought it was better prepared. The belief in being better prepared than other countries was strongly correlated with having right-leaning conservative views $(r=.56, p<.001)$. Specifically, among conservatives $(N=300), 54 \%$ thought the US was better prepared than other countries, $12.7 \%$ thought it was equally prepared, and $33 \%$ thought it was worse prepared. Among liberals $(N=682)$, in contrast, only $7.8 \%$ thought the US was better prepared than other countries, $4.8 \%$ thought it was equally prepared, and $87.4 \%$ thought it was worse prepared. Finally, we observed a negative correlation between the national belief in being better prepared and risk predictions for self $(r=-.24, p<.001)$ and others $(r=-.30, p<.001)$, and support of restrictive lockdown policies $(r=-.25, p<.001)$. That is, people who had the view that the US was better prepared than other countries to handle the pandemic also tended to be less supportive of early lockdown (March, 2020). We note that at the time these predictions were made, the sitting President was from the Republican party (Trump).

\begin{tabular}{|c|c|c|c|c|c|c|}
\hline & & Self: realistic risk & Other: realistic risk & Covid policy support & Political conservatism & US national preparedness \\
\hline Self: realistic risk & $\begin{array}{l}\text { Pearson's r } \\
\text { p-value }\end{array}$ & - & & & & \\
\hline Other: realistic risk & $\begin{array}{l}\text { Pearson's r } \\
\text { p-value }\end{array}$ & $\begin{array}{l}0.773 \cdots \\
<.001\end{array}$ & $\begin{array}{l}- \\
-\end{array}$ & & & \\
\hline Covid policy support & $\begin{array}{l}\text { Pearson's r } \\
\text { p-value }\end{array}$ & $\begin{array}{l}0.224 * * \\
<.001\end{array}$ & $\begin{array}{l}0.247 \cdots \\
<.001\end{array}$ & - & & \\
\hline Political conservatism & $\begin{array}{l}\text { Pearson's r } \\
\text { p-value }\end{array}$ & $\begin{array}{l}-0.188 * \cdots \\
<.001\end{array}$ & $\begin{array}{l}-0.241 \cdots \\
<.001\end{array}$ & $\begin{array}{l}-0.208 * \cdots \\
<.001\end{array}$ & $\begin{array}{l}- \\
-\end{array}$ & \\
\hline US national preparedness & $\begin{array}{l}\text { Pearson's r } \\
\text { p-value }\end{array}$ & $\begin{array}{c}-0.237^{\cdots \cdot} \\
<.001\end{array}$ & $\begin{array}{c}-0.301 \cdots \\
<.001\end{array}$ & $\begin{array}{c}-0.246^{\circ} \cdots \\
<.001\end{array}$ & $\begin{array}{l}0.557^{* * *} \\
<.001\end{array}$ & - \\
\hline
\end{tabular}

Note. $" p<.05, * p<.01, \cdots p<.001$

\section{Table 3}

Correlation matrix for Experiment $2(N=1202$, Prolific representative sample, USA), showing statistical associations between five key variables in the context of the COVID-19 pandemic.

Once again, we examined whether political orientation would moderate our primary finding of relative optimism. Reflecting the results from Experiment 1, a moderation analysis in Jamovi found no interaction between experimental condition and political orientation when retesting the best-case heuristic hypothesis in Experiment $2(Z=.83, p=.41)$. Despite lower general risk predictions among right-leaning conservatives than left-leaning liberals, the bestcase heuristic was equally prevalent on both sides of the right-left political spectrum. 


\section{Optimism: Relative vs. Absolute}

In terms of absolute risk levels, exploring the accuracy of risk predictions to actual infection rates by the end of the year, it is worth noting that the current predictions turned out to be pessimistic. According to one base-rate estimate relying on machine learning methods (Noh \& Danuser, 2021), about $10 \%$ of the adult American population got infected by the end of 2020 , which is much lower than predictions in the most "realistic scenario" of $37.6 \%$ in Experiment 1 and $36 \%$ in Experiment 2 (see Table 1). Thus, in the domain of covid infections during the first year of the pandemic, the current participants were optimistic in the relative sense, as predicted by our primary hypotheses, but also turned out to be pessimistic in absolute terms.

\section{Experiment 3: Conceptual Replication}

Experiment 1 and 2 was conducted in the specific context of infection rate predictions during the first wave of the covid pandemic. This raises the question of generalizability: If people rely on a best-case heuristic as a general prediction strategy, that should generate a broader pattern of relative optimism across different life domains.

To test this possibility, Experiment 3 was a pre-registered conceptual replication on Mechanical Turk ( $\mathrm{N}=840, \mathrm{PDF}$ : http://aspredicted.org/blind.php? $\mathrm{x}=\mathrm{ua3q} 9 \mathrm{x})$. This experiment focused on whether the best-case heuristic would generalize to estimates of when a COVID-19 vaccine would be publicly available in the US, and the unrelated domain of being in a happy romantic relationship five years into the future. Participants responded either to the vaccine questions or the relationship questions.

The data collection was conducted on the 15th of April (2020). After excluding 10 participants who failed a simple attention check, the final sample consisted of 840 participants $\left(M_{\text {age }}=38\right.$, years, $46 \%$ women). A sensitivity analysis showed that the final sample provided statistical power corresponding to an $80 \%$ chance to detect a main effect of $d=0.27$ or larger ( $p$ $<.05$, two-tailed) in each part of the experiment (vaccine and relationship predictions).

Procedure and measures. In Experiment 3, the participants predicted when they thought an effective COVID-19 vaccine would be publicly available, or how likely they thought it was that they would be in a happy relationship five years into the future. Using a similar experimental procedure as in Experiment 1 and 2, they first reported their prediction in the "most realistic case", and then were randomly assigned to make similar predictions either in an optimistic bestcase scenario or a pessimistic worst-case scenario on the next, separate screen. The participants responded either to the vaccine questions or the relationship questions, meaning that the total sample was divided between four groups in this study.

Vaccine prediction wording: "At what point do you think an effective vaccine against Corona will be publicly available?" (9-point scale, increasing in increments of four months: 2020 August; 2020 December; 2021 April; 2021 August; 2021 December; 2022 April; 2022 August; 2022 December; 2023 April or later). Relationship prediction wording: "How likely do you think it is that you will be in a happy relationship 5 years from now?" ( $0 \%$ likely - $100 \%$ likely, increasing in increments of 10).

\section{Best-case Heuristic 1: Vaccine Prediction}

\section{Results}

Experiment 3 took place in mid-April, 2020. As their most realistic estimate, the participants predicted that an effective vaccine against COVID-19 would be publicly available 14 months later $(M=14.01, S D=7.18)$, resembling June 2021. In their best-case scenario, they predicted that it would be available in 12 months $(M=11.81, S D=7.62)$, resembling April 2021. In their worst-case scenario, they predicted that it would take 25 months $(M=24.96, S D=$ 9.92), resembling May 2022. 
Extending our previous results, these estimates demonstrate another positive asymmetry, in which the distance from the "realistic" predictions to the best-case scenario are much smaller $(M=2.82, S D=5.45)$ than the distance from the realistic to the worst-case scenario $(M=11.56$, $S D=8.15)$. This difference was statistically significant, and the effect size was very large, $(t(414)=12.85, p<.001, d=1.26)$. Thus, when the participants were instructed to provide realistic predictions for when an effective COVID-19 vaccine would be available for public use, they were systematically leaning towards their best-case scenario for the future.

\section{Best-case Heuristic 2: Relationship Prediction}

To further investigate the generality of the best-case heuristic, as the novel and primary form of relative optimism in the current research, a different set of participants were asked to estimate their chances of being in a happy relationship five years from now. Logically, this prediction depends both on the likelihood of being in a relationship (vs. being single) and the likelihood of this relationship being a happy one (vs. neutral or unhappy). If people rely on the best-case heuristic as a generalized heuristic, beyond COVID-19 and global pandemics, we should observe a similar best-case asymmetry in this unrelated domain as well.

As their most realistic estimate, participants predicted that it was on average $71.5 \%$ likely $(S D=28.06)$ that they would be in a happy relationship five years later. In the best-case scenario, the participants predicted it to be $80.3 \%$ likely $(S D=24.55)$, and in the worst-case scenario, they thought it was only $46.6 \%$ likely $(S D=33.11)$. Like before, the distance from the "realistic" predictions to the best-case scenario was smaller $(M=9.81, S D=15.66)$ than the distance from the "realistic" predictions to the worst-case scenario $(M=25.83, S D=27.35)$. This difference was statistically significant, and the effect size was large $(t(422)=7.37, p<.001, d=0.72)$. Again, the estimates they believed to be realistic came much closer to their optimistic best-case scenario than to their pessimistic worst-case scenario, as judged by themselves, providing a conceptual replication of the best-case heuristic in a new and different life domain.
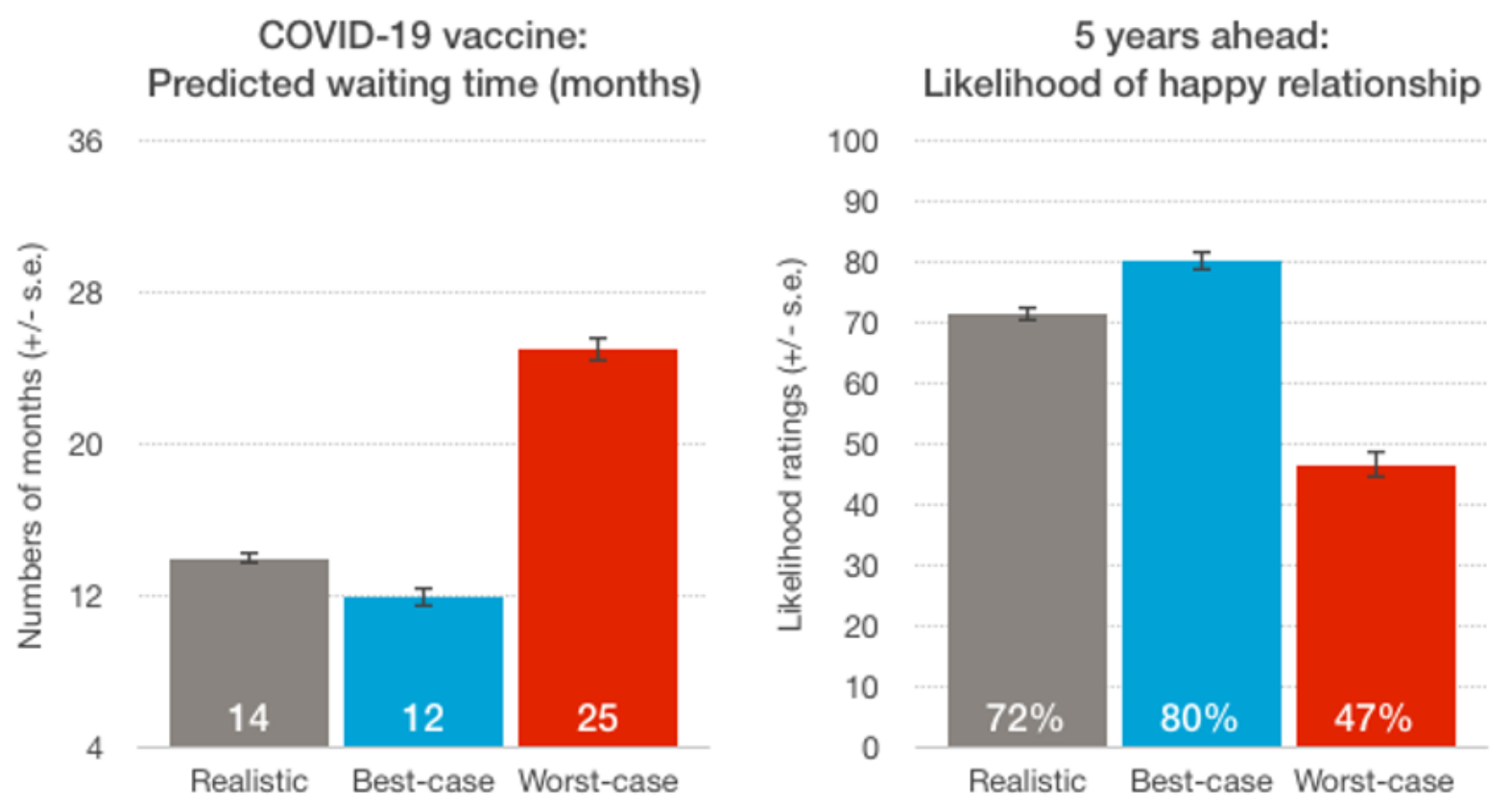

Figure 3: The best-case heuristic in different domains, Experiment 3

Experiment $3(N=840$, Mechanical Turk sample, USA): Providing a conceptual replication of the best-case heuristic from Experiment 1 and 2, the "realistic" estimates in Experiment 3 were positively skewed both in the case of predicted waiting time for a COVID-19 vaccine $(p<.001, d=1.26)$, and predicted likelihood of being in a happy relationship 5 years into the future $(p<.001, d=.72)$. That is, when participants tried to make their most realistic prediction in each of these domains, they were leaning towards their own best-case scenario for the future. 


\section{Optimism: Relative vs. Absolute}

Like before, our pre-registered primary hypotheses were concerned with relative optimism. As an explorative analysis for vaccine predictions, a comparison of "realistic" predictions with the subsequent timeline of actual availability shows a pessimistic pattern in absolute terms. Possibly reflecting the quickest successful vaccine development in the history of modern medicine (Saleh et al., 2021), which typically has taken 5-10 years according to a report from John Hopkins University, the first evidence-based vaccines against COVID-19 were publicly available to all adult Americans in all states by the end of April 2021 (CDC, 2022), just 12 months after Experiment 3 was conducted. This waiting time is in fact identical to the average "best-case" prediction by our participants, and shorter than their most "realistic" prediction (June, 2021). The prediction results for vaccines therefore provides a second example of coexisting optimism and pessimism in the covid context, when assessed either in relative or absolute terms, respectively.

In the case of future relationship satisfaction, however, the current data suggests that the participants were optimistic in both the relative and absolute sense. In this context, realism can be assessed by comparing the predictions in Experiment 3 with actual statistics on the prevalence of happy relationships. According to a nationally representative survey of Americans $(\mathrm{N}=4,860)$, the Pew Research Center (2021) reported that $69 \%$ of the adult population are in a committed relationship. Within the category of people in a relationship, however, their happiness with the relationship varies from positive to negative. A survey of Americans who were currently in a relationship $(\mathrm{N}=3,000)$, conducted by Harris Interactive and weighted to be nationally representative by age, gender and region, found that $75 \%$ considered themselves 'happy' in the relationship. Based on these numbers, we may estimate the conjunction probability of being in a happy relationship, $\mathrm{p}$ (In a relationship: .69 x Happy in relationship: .75), to be 52\%. When using this base-rate estimate as a rough benchmark for comparison, the actual probability of being in a happy relationship (52\%) appears to be much lower than the "most realistic" estimate of our study participants (71.5\%), and closer to their worst-case scenario prediction (46.6\%). Unlike covid infection rates and vaccine predictions, this comparison provides novel evidence that the best-case heuristic can also lead to optimistic bias in absolute terms. To be sure, the current baserate estimate is in itself uncertain, and input from different surveys may generate different estimates of the proportion of the population who are currently in a happy relationship.

\section{Experiment 4: Extension and Mechanism}

Based on the current evidence for a best-case heuristic, a critical reader could argue that there are at least three important limitations regarding interpretation and methodology. First, the experiments presented thus far have exclusively relied on a combined within/between-subjects design, in which all participants first report their "most realistic" prediction and then are asked to provide either their best-case or worst-case prediction. This design forces each participant to make two explicit judgments of the same event in a row, which might influence the second scenario predictions in an unintended manner. Second, if heuristics are to be understood as an effort-reducing simplification strategy (Shah \& Oppenheimer, 2008), it would be helpful to know whether it somehow requires more cognitive reflection to provide a pessimistic worst-case prediction than an optimistic best-case prediction. Third, if the best-case heuristic is a domaingeneral rule of thumb, it remains an open question whether it generalizes across different societal events, beyond the covid pandemic and social relationships.

To address these issues, Experiment 4 was a pre-registered extension in a nationally representative sample at Prolific Academic (N=583, PDF: https://aspredicted.org/5RZ LZL), leveraging important adjustments in experiment design. First, the new design was fully betweensubjects, with three separate prediction conditions: realistic, best-case, or worst-case. Second, hidden response time measures were activated in the online survey across all three conditions, 
enabling a comparison of whether it takes more time to generate a worst-case prediction than a best-case and "realistic" prediction. Third, in addition to relationship prediction as the primary outcome, we included a second exploratory outcome measure in which participants predicted whether the next president of the United States would come from the political party they identify the most with. Whereas the first and second addition can help provide novel evidence for or against a heuristic judgment process (i.e., a fully between-subjects design and response-time measures of the predictions), the third addition serves as an extended test of the generalizability of the best-case heuristic to a politically relevant future event (i.e., the next US presidential election).

The primary hypothesis in Experiment 4, was that "realistic" predictions of the probability of being in a happy relationship five years later would be significantly higher than the worst-case predictions, but not significantly lower than the best-case predictions. As a secondary analysis, we also tested the same hypothesis for probability judgments concerning the next presidential election in the US, and checked for response-time differences across the three conditions for each of the two prediction domains.

Procedure and measures. The data collection for Experiment 4 was conducted on the 9th of September (2022). Aiming for an attentive sample of 600 participants, we recruited 660 American online participants from the Prolific platform. After excluding 77 participants who failed a simple attention check at the end of the survey (providing the correct response to whether they had been asked to make predictions in a best-scenario, worst-case scenario, or realistic scenario), the final sample consisted of 583 attentive participants ( $M_{\text {age }}=37$, years; $44 \%$ single and $56 \%$ in a relationship; $49 \%$ women). A sensitivity analysis showed that the final sample provided statistical power corresponding to an $80 \%$ chance to detect a simple main effect of $d=0.29$ or larger ( $p<.05$, two-tailed) in two-condition comparisons.

Participants were randomly assigned to make future predictions in one of three conditions: "the most realistic case", an optimistic best-case scenario, or a pessimistic worst-case scenario. The headline instruction to each scenario prediction was kept the same as in the previous experiments. The main difference was that Experiment 4 was a fully between-subjects design, consisting of three separate conditions.

As the first outcome measure, participants responded to the relationship prediction question from in Experiment 3: "How likely do you think it is that you will be in a happy relationship 5 years from now?" (0-100\% likely) As the second outcome measure, presented separately on the next screen, participants were asked a question about the outcome of the next presidential election in their country, two years ahead: "How likely do you think it is that the next president of the United States will be someone from the political party you most identify with?" (0-100\% likely). The headline instruction above each prediction question was presented as one of the three following alternatives, depending on condition: "In the most realistic case:", "In the "best-case scenario" (an optimistic view):", or "In the "worst-case scenario (a pessimistic view):". Participants were kept in the same condition across both outcome measures.

At the end of the survey, participants responded to a simple attention check ("What did the questions in this survey ask you to consider? A best-case scenario; A worst-case scenario; The most realistic case"), before they reported demographic information (age, gender, current relationship status) and their political orientation on the same 7-point scale as before (1: Very liberal/left-leaning, 4: Centrist/moderate, 7: Very conservative/right-leaning).

\section{Best-case Heuristic 1: Relationship Prediction}

\section{Results}

Participants in the pessimistic worst-case condition predicted that it was only $44.7 \%$ likely to be in a happy relationship five years into the future $(S D=34.0)$. Participants in the 
realistic scenario condition predicted it was $62.5 \%$ likely $(S D=33.4)$, whereas participants in the optimistic best-case condition predicted it to be $64.3 \%$ likely $(S D=30.6)$. A one-way betweengroups ANOVA identified a significant difference between the three groups in how likely they thought it was to be in a happy relationship five years later, $\left(F(2,580)=21.14, p<.001, \eta^{2}=\right.$ .068). The proportion of participants who were currently in a relationship was equally balanced between the three conditions (worst-case: $57 \%$, realistic: $55 \%$, best-case: $56 \%$, ANOVA $p=$ .957).

In line with our primary and pre-registered hypothesis, Tukey post-hoc analyses indicated that predictions in the realistic condition were significantly higher than predictions in the worstcase condition $(62.5 \%$ vs. $44.7 \%, M$ difference $=17.7$ pp., $S E=3.27, p<.001)$, and that predictions in the best-case condition were not significantly higher than predictions in the realistic condition $(64.3 \%$ vs. $62.5 \%, M$ difference $=1.8$ pp., $S E=3.35, p=.854)$. Consistent with a best-case heuristic, these results reveal that even when participants were instructed to be as realistic as they could, their relationship predictions were positively skewed and statistically undistinguishable from similar predictions made in an optimistic best-case scenario.

As a non-registered analysis, aiming to provide a rough benchmark for assessing the statistical accuracy of these predictions, the base-rate estimation from Experiment 3 suggests that the actual probability of being in a happy relationship is currently around $52 \%$ in the United States. As such, the most "realistic" prediction from the study participants in Experiment 4 $(62.5 \%)$ were substantially higher than the current base-rate estimate of people being in happy relationships (52\%), suggesting optimism bias also in the absolute sense. Furthermore, we also note that the average "realistic" prediction of how likely it was to be in a happy relationship five years into the future $(62.5 \%)$ is higher than the total proportion of participants in Experiment 4 who currently were in any committed relationship at all (56\%).

\section{Best-case Heuristic 2: Election Prediction}

Before examining our second outcome, included as an exploratory measure in the same experiment design to ask about the next presidential election in the United States, we removed 106 participants who indicated that they did not consider themselves either a left-leaning liberal or a right-leaning conservative by reporting the number 4 on the 7-point scale of political orientation (midpoint heading: "Centrist/Moderate"). This exclusion left a final sample of 477 participants with political views either to the right or the left.

The results indicated that participants in the worst-case condition thought it was only $38.7 \%$ likely $(S D=25.4)$ that the next president of the United States would be someone from their own side of the political spectrum. Participants in the realistic scenario condition predicted that it was on average $55.1 \%$ likely $(S D=22.3)$, whereas participants in the best-case condition predicted it to be $56.3 \%$ likely $(S D=20.0)$. When conducting a one-way between-groups ANOVA, we identified a significant difference between the three groups in their election predictions $\left(F(2,474)=29.96, p<.001, \eta^{2}=.112\right)$.

Reflecting a similar pattern as the relationship predictions, Tukey post-hoc analyses found that predictions in the realistic condition were significantly higher than predictions in the worst-case condition $(55.1 \%$ vs. $38.7 \%, M$ difference $=16.4$ pp., $S E=2.51, p<.001)$, and that predictions in the best-case condition were not significantly higher than predictions in the realistic condition $(56.3 \%$ vs. $55.1 \%, M$ difference $=1.2$ pp., $S E=2.58, p=.888)$. Consistent with a best-case heuristic, these results reveal that even when participants were instructed to be as realistic as they could, their predictions for a future presidential election were positively skewed and statistically undistinguishable from similar predictions made in an optimistic bestcase scenario.

As a non-registered assessment of the expected group-level accuracy of these predictions, both left-leaning liberals and right-leaning conservatives thought it was more than $50 \%$ likely 
that their side would win the next presidential election in the most realistic scenario (liberals: $M$ $=54.7 \%, S D=21.4$, conservatives: $M=56.6 \%, S D=25.7)$, adding up to a self-favoring excess of subjective probability of 11,3 percentage points (i.e., a total sum of $111,3 \%$ rather than $100 \%$ ). As such, future optimism in "realistic" predictions was ideologically symmetric, where logically at least one of the two sides must be wrong in their average estimate.
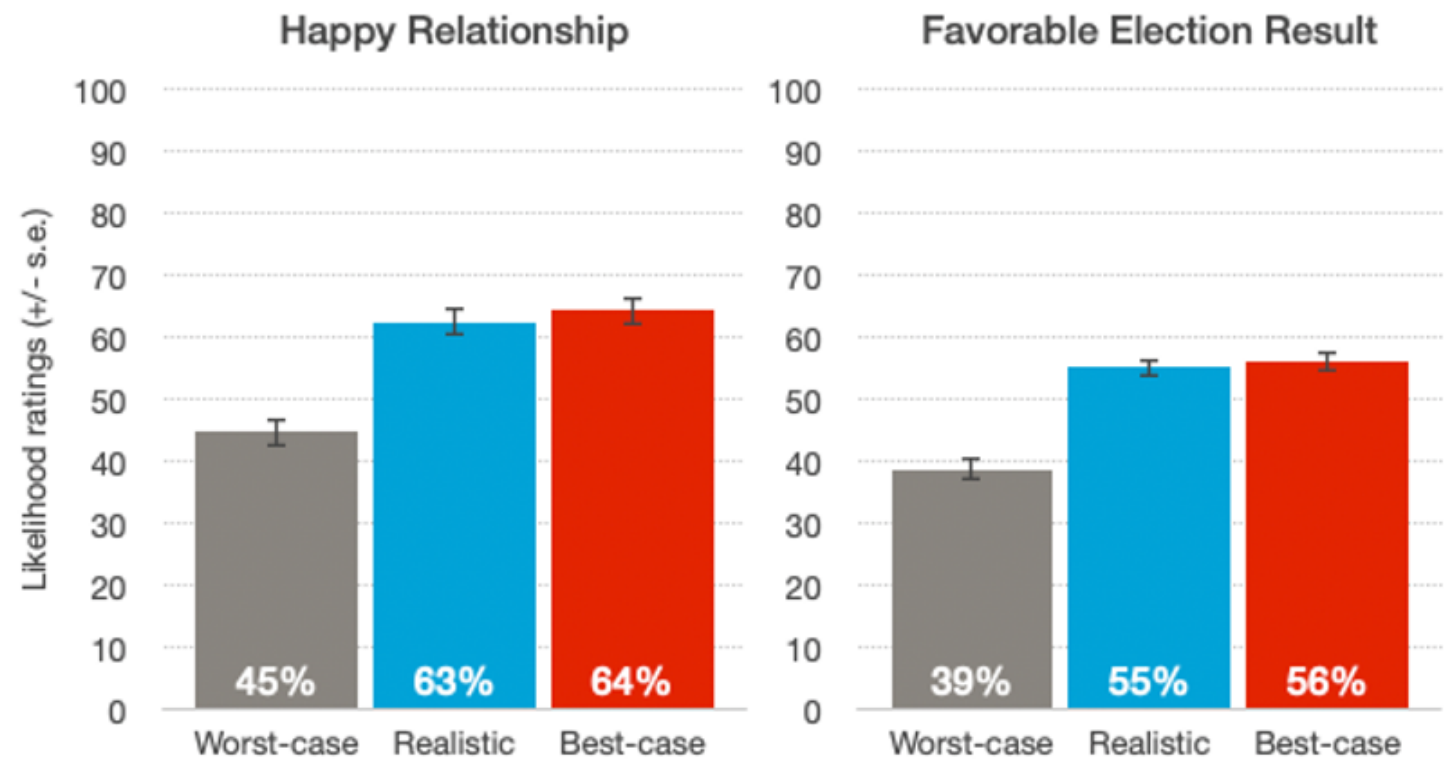

Figure 4: The best-case heuristic in relationships and politics, Experiment 4

Experiment $4(N=583$, Prolific representative sample, USA): Providing an extended replication of the best-case heuristic in a fully between-subjects design with three conditions (worst-case, realistic, best-case), the "realistic" estimates in Experiment 4 were positively skewed across two different life domains: The predicted likelihood of being in a happy relationship five years into the future $\left(p<.001, \eta^{2}=.112\right)$ and the predicted likelihood that one's own side of the right-left political spectrum would win the next presidential election in the US $\left(p<.001, \eta^{2}=.112\right)$. When participants were instructed to make their most realistic prediction, their average estimate in both life domains were practically identical to the average prediction made by participants in an optimistic best-case scenario.

\section{Response-Time Analysis}

To shed further light on the mechanism underlying the best-case asymmetry in "realistic" predictions, we examined response times across the three experimental conditions, operationalized as number of seconds from question presentation on screen to the last mouse click submitting the response to that specific question. One interpretation of this type of response-time data, is that heuristic thinking tends to be faster than reflective thinking, and relatedly, that longer response time is primarily a signal of decision conflict between opposing views or different courses of action (Evans et al., 2015). If "realistic" predictions are based on a heuristic best-case judgment, they should be faster. Alternatively, deviating from the optimistic default to consider a worst-case scenario should introduce higher decision conflict and thereby require longer response time.

Put differently, perhaps the act of making a pessimistic worst-case prediction requires the person to slow down a bit and "think twice" before responding. In line with this possibility, we found a consistent response-time difference across the three experimental conditions. Due to strong right-skewness of both of response-time variables, which is common for this type of data, they were $\log$ transformed with the base value of 10 for the statistical comparison below.

For the relationship prediction, participants in the worst-case condition spent on average 13.0 seconds $(S D=15.6)$ on their response, log-transformed mean $=1.01, S D=0.27$.

Participants in the "realistic" condition spent 9.3 seconds $(S D=17.9)$, log-transformed mean $=$ 
$0.84, S D=0.26$, whereas participants in the best-case condition spent 9.8 seconds $(S D=17.3)$, $\log$-transformed mean $=0.87, S D=0.26$. A one-way between-groups ANOVA identified a significant difference between the three groups in log-transformed response times, $(F(2,571)=$ $\left.22.76, p<.001, \eta^{2}=.074\right)$. Consistent with our heuristic account, Tukey post-hoc analyses revealed that predictions in the worst-case condition were significantly slower than similar predictions in the "realistic" condition ( $M$ difference $=0.17, S E=0.26, p<.001)$ and best-case condition ( $M$ difference $=0.14, S E=0.27, p<.001)$. Predictions in the "realistic" and best-case condition were faster than worst-case predictions, but they were not significantly different from each other $(M$ difference $=0.03, S E=0.27, p=.478)$.

For the political election prediction we observed a similar pattern. Participants in the worst-case condition spent on average 9.8 seconds $(S D=12.6)$, log-transformed mean $=0.91$, $S D=0.23$. Participants in the "realistic" condition spent 8.9 seconds $(S D=14.1), \log$ transformed mean $=0.85, S D=0.24$, whereas participants in the best-case condition spent 7.9 seconds $(S D=6.1)$, log-transformed mean $=0.85, S D=0.22$. A one-way between-groups ANOVA identified a significant difference between the three groups in log-transformed response times, $\left(F(2,466)=4.27, p=.015, \eta^{2}=.018\right)$. Consistent with our heuristic account, Tukey posthoc analyses revealed that predictions in the worst-case condition were significantly slower than similar predictions in the "realistic" condition ( $M$ difference $=0.63, S E=0.26, p=.036)$ and best-case condition ( $M$ difference $=0.67, S E=0.26, p=.030$ ). Predictions in the "realistic" and best-case condition were faster than worst-case predictions, but they were not significantly different from each other $(M$ difference $=0.003, S E=0.26, p=.998)$.

Although the group differences in response-time was somewhat smaller for election predictions than for relationship predictions, the general pattern was the same across both our two outcome measures. Seen as a whole, we consider this suggestive evidence for the proposed heuristic nature of the best-case asymmetry in future predictions.

\section{General Discussion}

Ranging from the context of a global public-health crisis, romantic relationships and political elections, the current research found robust evidence for two different kinds of relative optimism. First, during the early stage of the COVID-19 pandemic, we provided an extended replication of the classic finding of "unrealistic comparative optimism" (Weinstein, 1980; Shepperd et al., 2013): On average, people rated their own chance of getting infected by the Coronavirus by the end of the year as lower than the average person. Independent of the current studies, recent work has reported a similar findings of comparative optimism during the pandemic (e.g., Wise et al., 2020). Unlike previous research, however, we found the same pattern across three alternative scenarios for the future (realistic, best-case, and worst-case), in which participants consistently placed themselves at lower risk than others.

As the second form of relative optimism and our primary contribution, termed the bestcase heuristic, we repeatedly found that people made "realistic" risk estimates that were much closer to their best-case scenario than to their worst-case scenario. Importantly, the hypothesis of a best-case heuristic was supported across four experiments and more than 2,900 participants, which included both a high-powered direct replication and conceptual replications that went far beyond the covid context. Ranging from covid infection predictions and predicted waiting time for a vaccine, to the unrelated life domains of future relationship satisfaction and the outcome of the next presidential election in the United States, people were consistently leaning towards a personal best-case scenario when making their "most realistic" prediction.

\section{Heuristic Mechanism: Optimistic Substitution}

Our final experiment (Experiment 4) used a fully between-subjects design with three separate conditions, and provided important insights into the psychological mechanism. Both in 
the relationship and political election domain, the "most realistic" and best-case predictions were practically identical to each other and statistically indistinguishable, whereas worst-case predictions were much less favorable (as one would expect). In our view, this conflation of realistic and best-case predictions is highly consistent with our proposed mechanism of optimistic attribute substitution (e.g., Kahneman, 2003): When people are asked to make their most realistic prediction, they might unknowingly be responding to what they want to happen instead, because the best-case scenario comes first to mind.

Providing further support to the heuristic nature of the best-case asymmetry, Experiment 4 showed that pessimistic worst-case predictions were markedly slower in terms of response time, compared to similar predictions made in a realistic or best-case scenario (which were equally fast). As an exploratory result, this response-time finding suggests that people might need some extra time to slow down and think twice before they can deviate from the best-case default and generate a more balanced scenario prediction.

\section{Political Identity and Public Health}

Turning to the psychology of policy support in the covid context, a bipartisan majority of our participants supported public-health lockdown interventions, which according to some analyses could have saved approximately 36.000 lives if they were implemented at an earlier stage in the United States (Pei et al., 2020) and also enabled earlier re-opening of society and weaker impacts on the economy (Oliu-Barton et al., 2021).

Despite overlapping policy agreement in our study samples, the correlational analyses revealed that support of such interventions were associated both with political orientation and infection risk predictions. Providing some understanding of the minority who were opposed to public-health intervention at this early stage of the pandemic, the results suggest that a combination of relatively low risk perception, right-leaning political views, and belief in national superiority was part of the explanation. Indeed, political identities and cultural coalitions might have been shaping risk perceptions about infection rates and the need for public health interventions (see Calvillo et al., 2020). Despite the connection between political orientation and the general level of perceived risk, relative optimism in the form of the best-case heuristic appeared to be symmetric across the political spectrum.

\section{Limitations and Future Directions}

It is a notable limitation in the current research that the observational connection between public-health policy support, political orientation and risk perception do not enable causal inference. A second limitation is that the participants from all four experiments were recruited from a single cultural setting (USA). It remains a question for future research whether changes over time might indicate a causal relationship between these variables, whether identity-based resistance to public-health interventions might respond to targeted scientific information, and whether relative optimism is evident to the same extent in other cultural settings.

As a cautionary note for the theoretical interpretation of the main findings of relative optimism, such a pattern does not necessarily imply absolute optimism. In the literature on prediction errors, there is a central distinction between 'comparative' and 'absolute' unrealistic optimism at the group level (see Shepperd et al., 2013; 2015). Comparative optimism can mean judgments that are systematically favorable of oneself compared to other people, which is the classic (Weinstein, 1980) and much debated (Harris \& Hahn, 2011; Krizan \& Windschitl, 2007) form of unrealistic optimism. In the current research, we also made a broader contribution by studying people's internal alternatives for the future, comparing the relative position of their predictions in "realistic", best-case, and worst-case scenarios.

Claims about absolute optimism and optimistic bias, on the other hand, implies a more direct assessment of systematic deviation from external reality, normally requiring a comparison 
with a clear normative benchmark for what the correct answer should be (Baron, 2012) -- such as the statistical base-rate frequency. Indeed, in the current research, a critical reader may ask: "Bias, compared to what?" We did not formulate any hypothesis about the statistical accuracy of predictions before the current experiments were conducted, and so the reader should interpret such comparisons as exploratory. The best-case heuristic in future prediction was the most stable pattern across our four experiments, expressing a consistent form of relative optimism. In absolute terms, however, there was no consistent bias, and the results varied across domains. For relationship and election predictions in Experiment and 3 and 4, the predictions indicated absolute optimism. For covid-related predictions in Experiments 1-3, predictions reflected absolute pessimism compared to base-rate data, not optimism.

As a final note on limitations, the current experiments exclusively relied on likelihood judgments on numerical probability scales (0-100\%). The layperson interpretation of probability is debated (Gillies, 2016), and is itself a study topic in judgment and decision-making research (e.g., Keren \& Teigen, 2001; Reyna, 2004). Future research could apply a broader set of measurement formats to test for convergence or possible differences. More generally, future research may examine in what cases people maintain their hopeful leanings towards a best-case scenario, and in what cases they allocate equal attention to the less encouraging alternatives. For now, we conclude that relative optimism in general, and the best-case heuristic in particular, was a robust and replicable finding in the current research. 


\section{References}

Alicke, M. D. (1985). Global self-evaluation as determined by the desirability and controllability of trait adjectives. Journal of personality and social psychology, 49(6), 1621.

Altemeyer, B. (1981). Right-wing authoritarianism. Winnipeg: University of Manitoba Press.

Altemeyer, B. (1996). The authoritarian specter. Harvard University Press.

Bago, B., Rand, D. G., \& Pennycook, G. (2020). Fake news, fast and slow: Deliberation reduces belief in false (but not true) news headlines. Journal of Experimental Psychology: General, 149(8), 1608-1613. https://doi.org/10.1037/xge0000729

Baron, J. (2012). The point of normative models in judgment and decision making. Frontiers in Psychology, 3. https://doi.org/10.3389/fpsyg.2012.00577

Barr, M., Raphael, B., Taylor, M., Stevens, G., Jorm, L., Giffin, M., \& Lujic, S. (2008). Pandemic influenza in Australia: Using telephone surveys to measure perceptions of threat and willingness to comply. BMC Infectious Diseases, 8(1), 117. https://doi.org/10.1186/1471-2334-8-117

Baumeister, R. F., Maranges, H. M., \& Sjåstad, H. (2018). Consciousness of the future as a matrix of maybe: Pragmatic prospection and the simulation of alternative possibilities. Psychology of Consciousness: Theory, Research, and Practice, 5(3), 223-238. https://doi.org/10.1037/cns0000154

Buehler, R., Griffin, D., \& Peetz, J. (2010). The planning fallacy: Cognitive, motivational, and social origins. In Advances in experimental social psychology, Vol 43 (pp. 1-62). Academic Press. https://doi.org/10.1016/S0065-2601(10)43001-4

Bults, M., Beaujean, D. J., de Zwart, O., Kok, G., van Empelen, P., van Steenbergen, J. E., Richardus, J. H., \& Voeten, H. A. (2011). Perceived risk, anxiety, and behavioural responses of the general public during the early phase of the Influenza A (H1N1) pandemic in the Netherlands: Results of three consecutive online surveys. BMC Public Health, 11, 2. https://doi.org/10.1186/1471-2458-11-2

Capraro, V., Boggio, P., Böhm, R., Perc, M., \& Sjåstad, H. (2021). Cooperation and acting for the greater good during the COVID-19 pandemic.

Chambers, J. R., Windschitl, P. D., \& Suls, J. (2003). Egocentrism, event frequency, and comparative optimism: When what happens frequently is "more likely to happen to me". Personality and Social Psychology Bulletin, 29(11), 1343-1356.

Cooper, A. C., Woo, C. Y., \& Dunkelberg, W. C. (1988). Entrepreneurs' perceived chances for success. Journal of business venturing, 3(2), 97-108.

Covey, J. A., \& Davies, A. D. M. (2004). Are people unrealistically optimistic? It depends how you ask them. British Journal of Health Psychology, 9(1), 39-49. https://doi.org/10.1348/135910704322778713 
Ditto, P. H., Liu, B. S., Clark, C. J., Wojcik, S. P., Chen, E. E., Grady, R. H., ... \& Zinger, J. F. (2019). At least bias is bipartisan: A meta-analytic comparison of partisan bias in liberals and conservatives. Perspectives on Psychological Science, 14(2), 273-291.

Eidelman, S., Crandall, C. S., Goodman, J. A., \& Blanchar, J. C. (2012). Low-effort thought promotes political conservatism. Personality and Social Psychology Bulletin, 38(6), 808820.

Evans, A. M., Dillon, K. D., \& Rand, D. G. (2015). Fast but not intuitive, slow but not reflective: Decision conflict drives reaction times in social dilemmas. Journal of Experimental Psychology: General, 144(5), 951.

Fiagbenu, M. E., Proch, J., \& Kessler, T. (2021). Of deadly beans and risky stocks: Political ideology and attitude formation via exploration depend on the nature of the attitude stimuli. British Journal of Psychology, 112(1), 342-357.

Gillies, D. (2016). The propensity interpretation. The Oxford Handbook of Probability and Philosophy. Oxford: Oxford Handbooks.

Gollwitzer, A., Martel, C., Brady, W., Knowles, E., \& Van Bavel, J. (2020). Partisan Differences in Physical Distancing Predict Infections and Mortality During the Coronavirus Pandemic. https://doi.org/10.31234/osf.io/t3yxa

Harris, A. J., \& Hahn, U. (2011). Unrealistic optimism about future life events: a cautionary note. Psychological review, 118(1), 135.

Harris, A. J., de Molière, L., Soh, M., \& Hahn, U. (2017). Unrealistic comparative optimism: An unsuccessful search for evidence of a genuinely motivational bias. PloS one, 12(3), e0173136.

Herzog, S. M., \& Hertwig, R. (2009). The Wisdom of Many in One Mind: Improving Individual Judgments With Dialectical Bootstrapping. Psychological Science, 20(2), 231-237. https://doi.org/10.1111/j.1467-9280.2009.02271.x

Hoorens, V., Smits, T., \& Shepperd, J. A. (2008). Comparative optimism in the spontaneous generation of future life-events. British Journal of Social Psychology, 47(3), 441-451. https://doi.org/10.1348/014466607X236023

Hoorens, V., Van Damme, C., Helweg-Larsen, M., \& Sedikides, C. (2017). The hubris hypothesis: The downside of comparative optimism displays. Consciousness and Cognition, 50, 45-55.

Inbar, Y., Pizarro, D. A., \& Bloom, P. (2009). Conservatives are more easily disgusted than liberals. Cognition and Emotion, 23(4), 714-725. https://doi.org/10.1080/02699930802110007

Iyengar, S., Lelkes, Y., Levendusky, M., Malhotra, N., \& Westwood, S. J. (2019). The Origins and Consequences of Affective Polarization in the United States. Annual Review of Political Science, 22(1), 129-146. https://doi.org/10.1146/annurev-polisci-051117073034 
Jansen, L. A., Appelbaum, P. S., Klein, W. M., Weinstein, N. D., Cook, W., Fogel, J. S., \& Sulmasy, D. P. (2011). Unrealistic optimism in early-phase oncology trials. Irb, 33(1), 1.

Jost, J. T., Glaser, J., Kruglanski, A. W., \& Sulloway, F. J. (2003). Political conservatism as motivated social cognition. Psychological Bulletin, 129, 339-375.

Kahneman, D. (2003). Maps of bounded rationality: Psychology for behavioral economics. American economic review, 93(5), 1449-1475.

Keren, G. (1991). Calibration and probability judgements: Conceptual and methodological issues. Acta Psychologica, 77(3), 217-273.

Keren, G., \& Teigen, K. H. (2001). The probability-outcome correspondence principle: A dispositional view of the interpretation of probability statements. Memory \& Cognition, 29(7), 1010-1021.

Klein, W. M. (2002). Comparative risk estimates relative to the average peer predict behavioral intentions and concern about absolute risk. Risk, Decision and Policy, 7(2), 193-202.

Klein, R. A. (2014). Investigating variation in replicability: A "many labs" replication project. Social Psychology, 45(3), 142. https://doi.org/10.1027/1864-9335/a000178

Kriss, J. L., Hung, M., Srivastav. A., Black, C. L., Lindley, M. C., Lee, J. T., Koppaka, R., Tsai, Y., Lu, P., Yankey, D., Elam-Evans, L. D., \& Singleton, J. A. (2022) COVID-19 Vaccination Coverage, by Race and Ethnicity - National Immunization Survey Adult COVID Module, United States, December 2020-November 2021. MMWR Morb Mortal Wkly Rep 2022;71:757-763. DOI: http://dx.doi.org/10.15585/mmwr.mm7123a2

Krizan, Z., \& Windschitl, P. D. (2007). The influence of outcome desirability on optimism. Psychological bulletin, 133(1), 95.

Krizan, Z., \& Windschitl, P. D. (2007). The influence of outcome desirability on optimism. Psychological bulletin, 133(1), 95.

Kruger, J., \& Burrus, J. (2004). Egocentrism and focalism in unrealistic optimism (and pessimism). Journal of Experimental Social Psychology, 40(3), 332-340.

Kushner Gadarian, S., Goodman, S. W., \& Pepinsky, T. B. (2020). Partisanship, Health Behavior, and Policy Attitudes in the Early Stages of the COVID-19 Pandemic. SSRN Electronic Journal. https://doi.org/10.2139/ssrn.3562796

Mason, L. (2018). Uncivil Agreement: How Politics Became Our Identity (Illustrated edition). University of Chicago Press.

Moore, D. A., \& Healy, P. J. (2008). The trouble with overconfidence. Psychological Review, 115(2), 502-517. https://doi.org/10.1037/0033-295X.115.2.502

Newby-Clark, I. R., Ross, M., Buehler, R., Koehler, D. J., \& Griffin, D. (2000). People focus on optimistic scenarios and disregard pessimistic scenarios while predicting task completion times. Journal of Experimental Psychology: Applied, 6(3), 171-182. https://doi.org/10.1037/1076-898X.6.3.171 
Noh, J., \& Danuser, G. (2021). Estimation of the fraction of COVID-19 infected people in US states and countries worldwide. PloS one, 16(2), e0246772.

Oliu-Barton, M., Pradelski, B. S., Aghion, P., Artus, P., Kickbusch, I., Lazarus, J. V., ... \& Vanderslott, S. (2021). SARS-CoV-2 elimination, not mitigation, creates best outcomes for health, the economy, and civil liberties. The Lancet, 397(10291), 2234-2236.

Pew Research Center (2014). Ebola Worries Rise, But Most Are 'Fairly' Confident in Government, Hospitals to Deal With Disease. Pew Research Center - U.S. Politics \& Policy. https://www.pewresearch.org/politics/2014/10/21/ebola-worries-rise-but-mostare-fairly-confident-in-government-hospitals-to-deal-with-disease/

Pew Research Center (2020). Nearly Half of U.S. Adults Say Dating Has Gotten Harder For Most People in the Last 10 Years. https://www.pewresearch.org/socialtrends/2020/08/20/a-profile-of-single-americans/

Pei, S., Kandula, S., \& Shaman, J. (2020). Differential Effects of Intervention Timing on COVID-19 Spread in the United States. MedRxiv. https://doi.org/10.1101/2020.05.15.20103655

Phillips, J., Morris, A., \& Cushman, F. (2019). How we know what not to think. Trends in Cognitive Sciences, 23(12), 1026-1040. https://doi.org/10.1016/j.tics.2019.09.007

Redshaw, J., \& Suddendorf, T. (2016). Children's and Apes' Preparatory Responses to Two Mutually Exclusive Possibilities. Current Biology, 26(13), 1758-1762. https://doi.org/10.1016/j.cub.2016.04.062

Reyna, V. F. (2004). How people make decisions that involve risk: A dual-processes approach. Current directions in psychological science, 13(2), 60-66.

Rogers, T., Moore, D. A., \& Norton, M. I. (2017). The belief in a favorable future. Psychological Science, 28(9), 1290-1301.

Rose, J. P. (2010). Are direct or indirect measures of comparative risk better predictors of concern and behavioural intentions? Psychology and Health, 25(2), 149-165.

Rothman, A. J., Klein, W. M., \& Weinstein, N. D. (1996). Absolute and relative biases in estimations of personal risk 1. Journal of Applied Social Psychology, 26(14), 1213-1236.

Saleh, A., Qamar, S., Tekin, A., Singh, R., \& Kashyap, R. (2021). Vaccine development throughout history. Cureus, 13(7).

Shah, A. K., \& Oppenheimer, D. M. (2008). Heuristics made easy: an effort-reduction framework. Psychological bulletin, 134(2), 207.

Shepperd, J. A., Klein, W. M., Waters, E. A., \& Weinstein, N. D. (2013). Taking stock of unrealistic optimism. Perspectives on Psychological Science, 8(4), 395-411. 
Shepperd, J. A., Waters, E., Weinstein, N. D., \& Klein, W. M. P. (2015). A Primer on Unrealistic Optimism. Current Directions in Psychological Science, 24(3), 232-237. https://doi.org/10.1177/0963721414568341

Sjåstad, H., \& Baumeister, R. F. (2022). Fast optimism, slow realism? Causal evidence for a two-step model of future thinking. Pre-print/working paper retrieved at: https://psyarxiv.com/v6ksu/

Sjåstad, H., Skard, S., Thorbjørnsen, H., \& Norman, E. (2022). Self-serving optimism in hedonic prediction: People believe in a bright future for themselves but not for their enemies. Preprint/working paper retrieved at: https://psyarxiv.com/wgc $3 \mathrm{z}$

Stroop, J. R. (1932). Is the judgment of the group better than that of the average member of the group? Journal of Experimental Psychology, 15(5), 550-562. https://doi.org/10.1037/h0070482

Tanner, R. J., \& Carlson, K. A. (2009). Unrealistically optimistic consumers: A selective hypothesis testing account for optimism in predictions of future behavior. Journal of Consumer Research, 35(5), 810-822. https://doi.org/10.1086/593690

Tappin, B. M., \& McKay, R. T. (2017). The Illusion of Moral Superiority. Social Psychological and Personality Science, 8(6), 623-631. https://doi.org/10.1177/1948550616673878

Taylor, S. E., \& Brown, J. D. (1988). Illusion and well-being: a social psychological perspective on mental health. Psychological bulletin, 103(2), 193.

Tversky, A., \& Kahneman, D. (1974). Judgment under Uncertainty: Heuristics and Biases. Science, 185(4157), 1124-1131. https://doi.org/10.1126/science.185.4157.1124

Tybur, J. M., Merriman, L. A., Hooper, A. E. C., McDonald, M. M., \& Navarrete, C. D. (2010). Extending the Behavioral Immune System to Political Psychology: Are Political Conservatism and Disgust Sensitivity Really Related? Evolutionary Psychology, 8(4), 147470491000800420. https://doi.org/10.1177/147470491000800406

United States Coronavirus: 30,087,813 Cases and 547,325 Deaths - Worldometer. (n.d.). Retrieved March 15, 2021, from https://www.worldometers.info/coronavirus/country/us/

United States-Americans current relationship status, by age 2017. (n.d.). Statista. Retrieved March 15, 2021, from https://www.statista.com/statistics/669122/americans-currentrelationship-status-by-age/

Van Bavel, J. J. (2020). Perspective | In a pandemic, political polarization could kill people. Washington Post. Retrieved March 12, 2021, from https://www.washingtonpost.com/outlook/2020/03/23/coronavirus-polarization-politicalexaggeration/

Van Bavel, J. J., Baicker, K., Boggio, P. S., Capraro, V., Cichocka, A., Cikara, M., Crockett, M. J., Crum, A. J., Douglas, K. M., Druckman, J. N., Drury, J., Dube, O., Ellemers, N., Finkel, E. J., Fowler, J. H., Gelfand, M., Han, S., Haslam, S. A., Jetten, J., ... Willer, R. (2020). Using social and behavioural science to support COVID-19 pandemic response. Nature Human Behaviour, 4(5), 460-471. https://doi.org/10.1038/s41562-020-0884-z 
Van Bavel, J. J., \& Pereira, A. (2018). The Partisan Brain: An Identity-Based Model of Political Belief. Trends in Cognitive Sciences, 22(3), 213-224.

https://doi.org/10.1016/j.tics.2018.01.004

van Dolder, D., \& van den Assem, M. J. (2018). The wisdom of the inner crowd in three large natural experiments. Nature Human Behaviour, 2(1), 21-26.

Waters, E. A., Klein, W. M., Moser, R. P., Yu, M., Waldron, W. R., McNeel, T. S., \& Freedman, A. N. (2011). Correlates of unrealistic risk beliefs in a nationally representative sample. Journal of behavioral medicine, 34(3), 225-235.

Weinstein, N. D. (1980). Unrealistic optimism about future life events. Journal of Personality and Social Psychology, 39(5), 806-820. https://doi.org/10.1037/0022-3514.39.5.806

Wise, T., Zbozinek, T. D., Michelini, G., Hagan, C. C., \& Mobbs, D. (2020). Changes in risk perception and self-reported protective behaviour during the first week of the COVID-19 pandemic in the United States. Royal Society open science, 7(9), 200742. 